\title{
An Implementation Evaluation of A Comprehensive Sex Education Program in a Rural Indiana County
}

Lauren Estes ${ }^{1}$, Carolyn G. Meagher ${ }^{2}$, Monique Hensley ${ }^{3}$, Abby Hunt ${ }^{3}$, Cory Wuerch ${ }^{1}$, Mary

$$
\text { A. } \mathrm{Ott}^{2}
$$

${ }^{1}$ Indiana University School of Medicine; ${ }^{2}$ Indiana University School of Medicine, Department of Pediatrics, Division of Adolescent Medicine; ${ }^{3}$ Health Care Education and Training, Inc.

Background and Objective: Rural teen pregnancy is a significant public health problem, yet there are no evidence-based prevention programs (EBPs) developed for rural communities. Little is known about the process of implementing and adapting EBPs for rural communities. An implementation science framework is a useful way to methodologically examine EBPs replicated in new contexts. Specifically, the Evaluation, Preparation, Implementation, Sustainment (EPIS) framework was utilized for this project. The purpose of this study is to describe the challenges and sucesses in the implementation of EBPs in a rural community.

Project Methods: Youth RISE! is a federally funded, evidence based, community-wide sex education program implemented in Clinton County, Indiana. Qualitative interviews were conducted with key stakeholders involved in implementing the program $(n=22)$. Participants fulfilled diverse roles such as school administrators, teachers, local program partners, and the grantor. These interviews, conducted in-person or via telephone in 2018 and via Zoom in 2020, were subsequently transcribed, coded and analyzed using thematic analysis. Codes were developed from the EPIS implementation framework and an initial review of transcripts and field notes.

Results: Participants described the Youth RISE! Program as overwhelmingly successful. Qualitative analysis revealed three central themes of Community Readiness, Transparency, and Buy-In. Community readiness included timing, involvement of trusted community partners, and framing teen pregnancy as a public health issue rather than a values issue. Transparency included frequent communication between all parties, positive messaging, and a strong presence in the community. Buy-in included parent, teacher, and school administration support as well as maintaining equal partnerships between the community, school, and partner agencies.

Conclusion and Potential Impact: Contrary to popular beliefs, this rural community was willing to implement comprehensive sex education programs in its schools. This data may be used to inform future implementation of EBPs in rural communities. 